Published in final edited form as:

Clin Lymphoma Myeloma Leuk. 2018 January ; 18(1): e61-e67. doi:10.1016/j.clml.2017.10.006.

\title{
VcR-CVAD induction chemotherapy followed by maintenance rituximab produces durable remissions in mantle cell lymphoma: a Wisconsin Oncology Network study
}

\author{
J.E. Chang ${ }^{1}$, L.L. Carmichael ${ }^{2}$, K. Kim² ${ }^{2}$, C. Peterson ${ }^{3}$, D.T. Yang ${ }^{4}$, A.M. Traynor ${ }^{1}$, J. E. \\ Werndli $^{5}$, M. S. Huie ${ }^{1}$, T. A. McFarland ${ }^{1}$, M. Volk ${ }^{6}$, J. Blank ${ }^{6}$, N.S. Callander ${ }^{1}$, W.L. Longo ${ }^{1}$, \\ and B.S. Kahl ${ }^{7}$ \\ ${ }^{1}$ Department of Medicine, University of Wisconsin School of Medicine and Public Health and the \\ UW Carbone Cancer Center, Madison, WI, USA \\ ${ }^{2}$ Department of Biostatistics and Medical Informatics, University of Wisconsin, Madison, WI, USA \\ ${ }^{3}$ Aspirus Cancer Center, Wausau, WI, USA
}

Corresponding author: Julie E. Chang, MD, 4007 Wisconsin Institute of Medical Research, 1111 Highland Ave, Madison, WI 53705-2275, jc2@ medicine.wisc.edu, Phone: (608) 262-3970, Fax: (608) 262-4598.

Conflicts of interest:

$\begin{array}{ll}\text { Julie E. Chang } & \text { Research funding - Genentech. } \\ \text { Lakeesha L. Carmichael } & \text { None } \\ \text { KyungMann Kim } & \text { None } \\ \text { Christopher Peterson } & \text { None } \\ \text { David T. Yang } & \text { None } \\ \text { Anne M. Traynor } & \text { None } \\ \text { Jae E. Werndli } & \text { None } \\ \text { Michael S. Huie } & \text { None } \\ \text { Thomas A. McFarland } & \text { None } \\ \text { Michael Volk } & \text { None } \\ \text { Jules Blank } & \text { None } \\ \text { Natalie S. Callander } & \text { None } \\ \text { Walter L. Longo } & \text { None } \\ \text { Brad S. Kahl } & \text { Consulting - Genentech, Millennium. }\end{array}$

Author contributions: Julie Chang: Collected and analyzed data, prepared manuscript.

Lakeesha Carmichael: Analyzed data, assisted with manuscript preparation.

KyungMann Kim: Analyzed data, assisted with manuscript preparation.

Christopher Peterson: Enrolled research subjects, approved manuscript.

David Yang: Analyzed data, assisted with manuscript preparation.

Anne Traynor: Trial implementation and oversight, approved manuscript.

Jae Werndli: Trial design and implementation, data management.

Michael Huie: Enrolled research subjects, approved manuscript.

Thomas McFarland: Enrolled research subjects, approved manuscript.

Michael Volk: Enrolled research subjects, approved manuscript.

Jules Blanks: Enrolled research subject, approved manuscript.

Natalie Callander: Enrolled research subjects, approved manuscript.

Walter Longo: Enrolled research subjects, approved manuscript.

Brad Kahl: Designed clinical trial, analyzed data, prepared manuscript. 
${ }^{4}$ Department of Pathology, University of Wisconsin School of Medicine and Public Health, Madison, WI, USA

5UW Carbone Cancer Center, Madison, WI, USA

${ }^{6}$ Saint Vincent Regional Cancer Center, Green Bay, WI, USA

${ }^{7}$ Division of Medical Oncology, Washington University School of Medicine, St. Louis, MO

\section{Abstract}

Introduction-VcR-CVAD was developed as an intermediate-intensity induction regimen with maintenance rituximab (MR) to improve remission durations after first-line therapy for mantle cell lymphoma (MCL) in older and younger MCL patients.

Patients and Methods-Patients with previously untreated MCL received VcR-CVAD induction chemotherapy for 6 cycles (21-day cycles). Patients achieving at least a partial response received rituximab consolidation $\left(375 \mathrm{mg} / \mathrm{m}^{2} \times 4\right.$ weekly doses) and MR $\left(375 \mathrm{mg} / \mathrm{m}^{2}\right.$ every 12 weeks $\times 20$ doses). The primary end points were overall and complete response (CR), and secondary endpoints were PFS and overall survival (OS). Thirty patients were enrolled, with a median age of 61 years. There was an even distribution of patients $<60$ years and $\Varangle 60$ years. MIPI medium- or high-risk disease was present in 60\%. Overall response rate observed was $90 \%$ (77\% CR/unconfirmed CR). After a median follow-up of 7.8 years, the 6-year PFS and OS were 53\% and $70 \%$, respectively. There was no difference in 6-year PFS or OS between the younger (age $<60$ ) and older (age 60 ) subgroups. In a univariate analysis, LDH when analyzed for interaction with age had a significant effect on PFS outcomes at 6 years. There were no pre-treatment variables determined to have a significant effect on OS outcomes at 6 years.

Conclusions-Long-term outcomes with VcR-CVAD are comparable with more intensive inductions and consolidation approaches. MCL is biologically heterogeneous and durable remission can be achieved with intermediate intensity therapy. Maintenance rituximab appears to contribute to these excellent outcomes.

\section{Keywords}

Mantle cell lymphoma; bortezomib; rituximab; non-Hodgkin lymphoma

\section{Introduction}

Mantle cell lymphoma (MCL) is a subtype of non-Hodgkin lymphoma (NHL) with biological characteristics intermediate between indolent and aggressive histologies. Although MCL has historically been treated with regimens administered for treatment of other high-grade non-Hodgkin lymphomas (NHLs), chemotherapy is not curative for MCL, and the short median progression-free survival (PFS) of 16-17 months with R-CHOP (rituximab, cyclophosphamide, doxorubicin, vincristine, prednisone) is substantially shorter than that observed in other subtypes of indolent NHLs. ${ }^{1,2}$ The median age of MCL diagnosis is 64 years, ${ }^{3}$ which increases the likelihood of concurrent co-morbidities that may further limit the tolerability of treatment-related toxicities. 
Due to the limited benefit observed with R-CHOP chemotherapy, a modified hyper-CVAD regimen was developed as an intermediate-intensity regimen to escalate the dose-intensity of induction chemotherapy, yet with a toxicity profile more tolerable than intensive approaches (i.e., hyper-CVAD chemotherapy, autologous stem cell transplant consolidation). ${ }^{4-6}$ Previous experience with rituximab + modified hyper-CVAD followed by maintenance rituximab through the Wisconsin Oncology Network (WON) had shown promising improvement in median PFS to 37 months in previously untreated MCL. ${ }^{7}$ With the emergence of bortezomib as an active single-agent in MCL, this agent was incorporated into a modified rituxuimab + hyper-CVAD induction, and termed VcR-CVAD. Outcomes with VcR-CVAD induction followed by 5 years of maintenance rituximab have previously been reported, with a 3 -year PFS of $63 \% .{ }^{8}$ Longer-term survival outcomes and toxicities are now reported.

\section{Material and methods}

\section{Patients}

This was prospective study performed through the Wisconsin Oncology Network (WON), a cooperative group consisting of the University of Wisconsin Carbone Cancer Center and several affiliated community-based practices. Eligible patients were age $\geq 18$ with a histologically confirmed diagnosis of MCL and measurable or evaluable disease. Patients were previously untreated except for up to 1 cycle of CHOP or CHOP-like therapy. Patients were required to have an ECOG performance status $\mathcal{2}$ and adequate serologic parameters (neutrophils $\geq 1500 / \mathrm{mm}^{3}$, platelets $\geq 100,000 / \mathrm{mm}^{3}$, serum creatinine $\mathcal{2} \mathrm{mg} / \mathrm{dL}$, serum bilirubin $\_\mathrm{mg} / \mathrm{dL}$, and AST $\_.5$ times the laboratory upper limit of normal). Low blood counts were not exclusionary if related to splenomegaly or disease replacement of bone marrow. Patients were ineligible if they had known central nervous system involvement by lymphoma, were pregnant or breast-feeding, or had an active second malignancy requiring radiation or chemotherapy treatments. Additional exclusionary criteria were known HIV infection, chronic or active hepatitis B infection, or NYHA class III or IV heart failure. The study was approved by the Human Subjects Committee at the University of Wisconsin and by the Institutional Review Board at each participating WON institution. All patients signed an informed consent document describing the investigational nature of the proposed treatment.

\section{Treatment}

The VcR-CVAD (bortezomib/Velcade ${ }^{\mathrm{TM}}$, rituximab, hyper-fractionated cyclophosphamide, vincristine, doxorubicin, dexamethasone) regimen has previously been described. ${ }^{9}$ Briefly, patients received 6 cycles of VcR-CVAD induction therapy (28-day cycles): rituximab 375 $\mathrm{mg} / \mathrm{m}^{2} \mathrm{IV}$ day 1 , bortezomib $1.3-1.5 \mathrm{mg} / \mathrm{m}^{2} \mathrm{IV}$ days $1 \& 4$, cyclophosphamide $300 \mathrm{mg} / \mathrm{m}^{2}$ IV every 12 hours days 1-3 (6 doses), vincristine 1-2 mg IV day 3, doxorubicin $50 \mathrm{mg} / \mathrm{m}^{2}$ continuous infusion over days $1-2$ (total dose $50 \mathrm{mg} / \mathrm{m}^{2}$ ), and dexamethasone $40 \mathrm{mg}$ orally days 1-4. Due to high rates of sensory neuropathy at the initial starting doses of bortezomib $1.5 \mathrm{mg} / \mathrm{m}^{2}$ and vincristine $2 \mathrm{mg}$, bortezomib dosing was reduced to $1.3 \mathrm{mg} / \mathrm{m}^{2}$ after the first 7 patients were enrolled, and vincristine dosing was reduced to $1 \mathrm{mg}$ after 14 patients were enrolled. Induction therapy was followed by 4 weekly doses of rituximab $375 \mathrm{mg} / \mathrm{m}^{2} / \mathrm{dose}$ as consolidation therapy, followed by a single dose of rituximab $375 \mathrm{mg} / \mathrm{m}^{2}$ every 3 months for 
a total of 5 years of maintenance therapy (total of 20 maintenance doses of rituximab).

Pegfilgrastim ( $6 \mathrm{mg} \mathrm{SQ}$ ) or G-CSF ( $5 \mathrm{mcg} / \mathrm{kg} /$ day) were required beginning on day 5 or 6 of the cycle. All appropriate supportive care measures were permitted throughout treatment including tumor lysis syndrome prophylaxis, transfusion support and antibiotics. Protocol therapy was discontinued in the event of disease progression, unacceptable toxicity, or physician and/or patient discretion. Toxicities were reported in accordance with the Common Terminology Criteria for Adverse Events (CTCAE), version 3.0. ${ }^{10}$

\section{Patient evaluation and response criteria}

Patients were assessed for response by CT imaging after cycles 2, 4 and 6 of induction VcRCVAD, following consolidation therapy, every 6 months during the maintenance phase, and annually following completion of therapy. Responses were defined by the 1999 International Working Group criteria. ${ }^{11}$

\section{Statistical considerations}

The primary endpoint of this study was the observed overall response (OR) and complete response (CR) to the VcR-CVAD regimen at the completion of induction therapy. A twostage design was undertaken to test the null hypothesis that a true probability of OR is at most 0.7 versus the alternative hypothesis that it is at least 0.9 . The significance level of this test is 0.10 with a power of 0.9 . Initially, 15 evaluable patients were to be assessed for response, with plans to terminate enrollment early if there were 11 or fewer objective responses observed. An additional 15 evaluable patients were to be enrolled if there were 12 or more objective responses observed in the first stage of the study.

Secondary endpoints included determination of PFS and OS, and assessment of tolerability and toxicity associated with the treatment. PFS was calculated from the date of first VcRCVAD administration until progression of disease or death from any cause. OS was calculated from the date of first VcR-CVAD administration until death from any cause.

The survival distribution for PFS and OS was estimated using the Kaplan-Meier method. Cox proportional hazard regression models and log-rank tests were used to assess whether baseline characteristics were predictive of PFS and OS.

\section{Results}

\section{Clinical characteristics}

Between July 2005 and May 2008, 30 patients were enrolled from four institutions. Details of patient characteristics have been published previously. ${ }^{8}$ Briefly, the median age for enrolled patients was 61 years, with $80 \%$ men. Half of enrolled patients were age $<60$ years, and half were age $\geq 60$ years. All patients had advanced stage disease, and six patients had blastoid morphology. The MIPI risk score indicated medium- or high-risk disease in $60 \%$ of patients. 


\section{Response}

All 30 enrolled patients were evaluable for response. Three patients experienced progression during induction therapy, and the remaining 27 patients experienced an objective response (ORR 90\%, 95\% confidence interval [CI] 73-98\%). Twenty-three patients achieved a CR or unconfirmed CR (77\%, 95\% CI 59-88\%). Three of four patients with a partial response after induction chemotherapy improved to a CR during maintenance rituximab.

\section{Progression-free and overall survival}

After a median follow-up of 7.8 years among surviving patients, the median PFS was 8.14 years (95\% CI 2.81-not reached) and the median OS has not been reached (Figure 1). Sixyear estimates of PFS and OS are 0.53 and 0.70 , respectively (Table 1). No relapses were observed beyond 6 years.

Fifteen patients $(50 \%)$ remains alive and in continuous first-remission, with an even distribution of patients age $<60$ years $(n=15)$ and $\ 60$ years $(n=15)$. Ten deaths have occurred; five related to progressive MCL, 3 related to complications of allogeneic transplant, and 2 related to second malignancies (lung cancer and T-cell non-Hodgkin lymphoma).

\section{Univariate analyses}

The 6-year PFS and OS by pre-treatment variables are shown in Table 1. Given the equal distribution of patients among younger and older subgroups, each pre-treatment variable was evaluated for interaction with age. The pre-treatment variable that was found to have a significant effect on the PFS outcomes at 6 years included LDH group by age group ( $<60$ vs. $\checkmark 60$ years). There were no pre-treatment variables that were determined to have a significant effect on OS outcomes at 6 years, although there was a trend towards significance between age group and elevated LDH.

Age-There was no difference in PFS or OS by age among the older ( $\ 60$ years) and younger ( $<60$ years) subgroups (Table 1 ). The median PFS was 8.14 years $(95 \%$ CI 2.2 -not reached) for the younger subgroup and 4.65 years (95\% CI 2.51-not reached) for the older age group $(\mathrm{p}=0.703$, Figure 1$)$.

MIPI score-The observed difference in PFS and OS between the three MIPI groups was not significant (Table 1 ). The association between age group ( $<60$ vs. $\succ 60$ years) and MIPI level on PFS showed no significant interaction $(\mathrm{p}=0.198)$. Similarly, there was no interaction between age group and MIPI on OS ( $\mathrm{p}=0.124)$.

Blastoid histology-Of 6 patients with blastoid histology, 3 remain in continuous remission on long-term follow-up. Blastoid histology was not significantly associated with worse PFS or OS on long-term follow-up (Table 1). The association between blastoid histology status and age group on PFS and OS were assessed and no significant interactions were found. 
LDH level-No difference in PFS was observed between those patients with an elevated versus normal LDH. However, there was a significant interaction $(\mathrm{p}=0.001)$ between age group and LDH level for PFS (Table 1). Among patients with an elevated LDH, younger patients had improved PFS compared with older patients (relative risk $[R R]=0.21,95 \% \mathrm{CI}$ $0.05-0.81, \mathrm{p}=0.024)$. Among patients with a non-elevated $\mathrm{LDH}$, the younger patients had less favorable PFS compared with the older patients ( $R R=20.64,95 \%$ CI 2.04-209.17, $\mathrm{p}=0.049$ ).

There was a trend towards a significant interaction between age group and LDH level for OS $(\mathrm{p}=0.053)$. Among patients with an elevated $\mathrm{LDH}$, there was a trend toward the younger age group having improved OS ( $\mathrm{RR}=0.25,95 \%$ CI $0.05-1.28, \mathrm{p}=0.096)$. Among patients with a non-elevated LDH, there was no significant difference in OS between the younger and older group $(\mathrm{p}=0.225)$.

Other baseline characteristics-There was no association between PFS and OS and the additional baseline characteristics of $\beta_{2}$-microglobulin, ECOG performance status, number of extranodal sites, and Ki-67 proliferative index. In addition, no interaction was found between these variables and age in predicting PFS and OS.

\section{Multivariate analysis}

In a multivariate analysis, the baseline factors of ECOG performance status and increase in number of extranodal sites were associated with overall survival. There was a trend toward an increase in ECOG PS by one being associated with worse OS ( $R R=5.35,95 \%$ CI 1-28.75, $\mathrm{p}=0.051$ ) and an increase in number of extranodal sites by 1 was associated with better OS $(\mathrm{RR}=0.06,95 \% \mathrm{CI} 0.01-0.66)$. The other baseline characteristics were not significantly associated with OS.

\section{Treatment tolerability}

Tolerability of VcR-CVAD induction has been previously published. ${ }^{8}$ The median cycles of MR received was 10.5 (median 21 months), with a dose intensity of MR of 59\% for those receiving at least 1 cycle. Six patients (20\%) were able to receive all 20 doses of planned MR. Fifteen (50\%) patients received 11-20 doses of MR. Among the 15 patients in longterm first remission, the median number of MR doses was 12 . This compared with median number of MR doses of 5 among patients not achieving long-term remission (included 4 patients who never started MR).

\section{Toxicity}

Acute toxicities to the regimen have been previously published. ${ }^{8}$ Since that publication, there have been not cases of treatment-related myelodysplasia or acute leukemia reported. The 2 events of secondary malignancies post-therapy (T-cell NHL and lung cancer) were not attributable to protocol therapy.

Hypogammaglobulinemia with recurrent infections occurred in 10 patients (33\%), including 4 patients who required $>6$ months of gamma globulin support. Among patients with hypogammaglobulinemia, two serious infectious events were observed (pneumococcal 
sepsis and recurrent Clostridium difficile infection). Eight patients with hypogammaglobulinemia experienced recurrent grade 1-2 respiratory infections.

Six events of delayed neutropenia occurred during MR in 5 patients. All responded to GCSF and did not limit further rituximab administration.

\section{Discussion}

Therapy for MCL has evolved significantly in the last 15 years, prior to which time outcomes were poor with standard R-CHOP chemotherapy. ${ }^{1,2}$ Although improvements in PFS were reported with front-line therapy using intensive regimens such as R-hyper-CVAD or R-maxi-CHOP and the use of autologous stem cell transplant (ASCT) as consolidation therapy, these approaches are not feasible for many adult patients due to toxicity concerns. 4,12 For example, the long-term remissions reported in the single-institution experience with R-hyper-CVAD could not be replicated in the multi-institution setting, where approximately $40-60 \%$ of patients were unable to complete protocol therapy. ${ }^{13,14}$ Similarly, approaches with consolidative autologous transplant typically limited enrollment to patients under the age of $65 \cdot \cdot^{4-6}$

Aside from intensive therapies limited to younger patients, advancements in MCL therapy in older adults have included the development of intermediate intensity regimens and/or incorporation of maintenance therapies. The experience previously published with Rmodified hyper-CVAD followed by 2 years of maintenance rituximab reported a median PFS of 37 months and therapy that was well tolerated. ${ }^{15}$ The subsequent development of VcRCVAD was aimed at further improving depths of remission with the addition of bortezomib to induction and extending the duration of maintenance rituximab, with the resulting improvement of 3-year PFS to $>60 \%{ }^{8}$ The European MCL Network established the benefit of maintenance rituximab in significantly improving PFS after a non-intensive induction of FCR (fludarabine, cyclophosphamide, rituximab) versus R-CHOP. ${ }^{15}$

The enrolled population in this trial is representative of a typical MCL population, with half

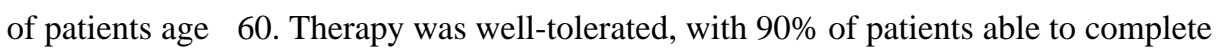
induction therapy, with the only reason for not completing induction therapy being progressive disease in 3 patients. ${ }^{8}$ Maintenance rituximab was associated with a reasonably high rate of hypogammaglobulinemia leading to premature discontinuation of MR during a planned 5 year course of maintenance.

Optimal duration of maintenance therapy in MCL remains unclear. The initial report with Rmodified-hyper-CVAD and MR by the Wisconsin Oncology Network limited the duration of maintenance to 2 years. The reported median PFS of 37 months with R-modified-hyperCVAD and MR demonstrated less durable disease controlled compared with VcR-CVAD. ${ }^{16}$ Whether the improved outcomes with VcR-CVAD versus R-modified-hyper-CVAD are due to the addition of bortezomib or extended duration of MR is uncertain. However, 2 large randomized intergroup trials have reported overall survival advantages with use of more extended courses of MR beyond the standard 2-year course of MR established in indolent NHLs. ${ }^{15,17}$ The European MCL Network reported a significant improvement in overall 
survival when an indefinite course of MR was given after a non-intensive R-CHOP induction compared with maintenance interferon-a (4-year survival $87 \%$ versus $63 \%, \mathrm{p}=0.005) .{ }^{15}$ At 4-years of follow-up, only $28 \%$ of patients had stopped MR prematurely. ${ }^{15}$ The experience in the LyMa trial found an overall survival advantage with 3-years of MR after a cytarbinecontaining induction and consolidative ASCT (4-year EFS 78.9\% with MR versus $61.4 \%$ without MR, $p=0.0012) .{ }^{17}$ It is possible that the extended course of MR in this study was important for maintaining durability of remissions. However, prolonged maintenance therapy also was associated with increased risk of hypogammaglobulinemia and associated complications. Further research designed to identify the optimal duration of maintenance therapy in MCL is warranted.

Importantly, no relapses were observed beyond 6 years of follow-up. Although it is probable that a continuous pattern of relapse will be observed as previously reported on long-term follow-up with R-hyper-CVAD and R-maxi-CHOP with ASCT, ${ }^{18,19}$ the impressive duration of first remission with this intermediate-intensity regimen is comparable with that reported from other intensive induction approaches only tolerable in younger and fitter patients (Table 2). Notably, there was no difference in PFS or overall survival between younger patients (<age 60) versus older patients (age 260 ). Although the number of patients with blastoid morphology was small $(n=6)$, these patients did not experience worse survival outcomes, and 3 of these patients remain in continuous first long-term remission.

E1405 was developed as a follow-up study to evaluate VcR-CVAD in the multicenter setting. It is possible that Patients in E1405 were permitted to undergo ASCT as consolidation after VcR-CVAD induction. This design was performed due to the hypothesis that induction VcR-CVAD may be a primary contributor to the observed durable remissions, and that younger patients receiving VcR-CVAD may achieve further improvement in PFS if consolidated with ASCT. In patients not receiving ASCT, 2 years of maintenance rituximab (MR) was administered. Initial reporting of E1405 showed 3 year PFS of 67\% among patients receiving MR, and therapy was well-tolerated. No significant difference in PFS or OS was observed between patients treated with MR and ASCT. Longer-term follow is planned to determine if the promising durable remissions with VcR-CVAD can be replicated in the cooperative group setting.

An important question remains as to how VcR-CVAD may fit into the current treatment paradigm for MCL. One important aspect of VcR-CVAD is demonstration of the proof of principle that intensive therapy approaches in induction and/or consolidation may not be required to achieve durable remissions with first-line therapy in MCL. This is important given that a substantial number of patients may not clearly fit into the current dichotomous treatment paradigm of intensive versus non-intensive therapy approaches. A common scenario faced by clinicians is an older adult in the $6^{\text {th }}$ or $7^{\text {th }}$ decade of life in good health but in whom toxicity with ASCT may not have an acceptable risk. However, this type of patient could tolerate therapy that is intensified beyond a standard R-CHOP or bendamustine-based induction. This common scenario illustrates the need for either: 1.) Ongoing use and development of intermediate intensity regimens, 2.) Rational incorporation of novel agents into an induction or maintenance, or 3.) Better prospective means of identifying patients who may have durable benefit from less intensive therapy. 
E1411 was developed to investigate the incorporation of novel agents into a standard induction and maintenance approach. Patients were randomized upfront to receive bendamustine + rituximab (BR) induction versus $\mathrm{BR}+$ bortezomib followed by maintenance with either single-agent rituximab versus rituximab + lenalidomide. Accrual to this trial was completed in 2016, and results are pending. Other approaches have included incorporation of the highly active Bruton's tyrosine kinase inhibitor ibrutinib in front-line therapy of MCL. ${ }^{20}$ Experience with BR chemotherapy combined with ibrutinib during chemotherapy followed by continuous ibrutinib dosing until progression was reported in multiple NHL histologies, including $17 \mathrm{MCL}$ patients (5 previously untreated). The OR among the MCL patients was 94\% (76\% CR), and the median PFS has not been reached. ${ }^{20}$ An ongoing trial (NCT0177684) is investigating outcomes in previously untreated MCL treated with a standard bendamustine and rituximab induction combined with ibrutinib.

Minimal residual disease testing is also being investigated as a means to better understand the intensity of therapy that may be required to achieve durable disease remissions. A potential advantage of MRD testing is that it may not require upfront commitment to a pathway of treatment intensity, but instead tailor intensity (i.e., need for consolidative ASCT) based on therapy response. A report of CALGB 50403 investigated prognostic implications of MRD status using PCR-based techniques in 49 MCL patients treated with intensive induction chemotherapy and consolidative ASCT for whom sequential MRD samples were available. ${ }^{21}$ Patients with early eradication of MRD following 2 cycles of intensive induction therapy had significantly improved PFS ( $\mathrm{p}=.017)$. None of the patients who achieved MRD-negative status post-induction $(n=15)$ had relapsed after a median of 3.3 years of follow-up. ${ }^{21}$

Pott et al described clinical outcomes as it relates to MRD status in 2 large international phase III trials of the European MCL Network in which MRD was a secondary endpoint. ${ }^{22}$ The 2 trials included both younger patients treated with a more intensive induction and ASCT (MCL Younger) and older patients (MCL Elderly) treated with a less intensive induction (R-FC versus R-CHOP) followed by rituximab or interferon maintenance. MRD data and a documented clinical remission after induction was available in 156 patients. Patients achieving a MRD-negative clinical remission after induction had an $87 \%$ chance of ongoing remission at 2 years compared with $61 \%$ of patients with MRD-positive clinical remission $(\mathrm{p}=.004) .{ }^{23}$ Sustained MRD negativity during maintenance therapy was also predictive of outcome. In the MCL Elderly trial, the response duration at 2 years was $76 \%$ in those with sustained MRD-negative status, compared with $36 \%$ of those with persistent residual disease by MRD analysis $(\mathrm{p}=.015){ }^{23}$

These compelling data regarding the prognostic value of MRD status post-induction therapy in MCL are the basis for the intergroup study EA4151. In this trial, patients are allowed to receive any induction regimen, with risk stratification to either consolidation ASCT versus MR based on the MRD status by next generation sequencing post-induction. Patients in a partial remission or with MRD positivity post-induction will go on to receive consolidative ASCT. In patients achieving a MRD-negative CR, patients will be randomized to ASCT versus MR. This study will provide valuable insight into the ability of MRD testing to 
prospectively identify patients who may benefit from less intensive therapy with preserved outcomes.

\section{Conclusion}

VcR-CVAD is a moderate-intensity regimen with favorable 6-year PFS and a toxicity profile that allowed tolerability even in a population with $50 \%$ of enrolled patient age $₹ 60$. An important lesson illustrated by VcR-CVAD is that long-term remissions are achievable in some patients without intensive inductions or consolidation. The development of novel agents with activity in MCL and prognosticating information (i.e., MRD testing) will likely further shape induction and maintenance strategies with a toxicity and activity profile that may be more favorable than VcR-CVAD.

\section{Acknowledgments}

Funding: This work was supported by the National Institutes of Health [P30 CA14520], Millennium Pharmaceuticals, Inc., and the University of Wisconsin Forward Lymphoma Research Fund.

\section{References}

1. Howard OM, Gribben JG, Neuberg DS, et al. Rituximab and CHOP induction therapy for newly diagnosed mantle-cell lymphoma: molecular complete responses are not predictive of progressionfree survival. J Clin Oncol. 2002; 20:1288-94. [PubMed: 11870171]

2. Robak T, Huang H, Jin J, et al. Bortezomib-based therapy for newly diagnosed mantle-cell lymphoma. N Engl J Med. 2015; 372:944-53. [PubMed: 25738670]

3. Zucca E, Roggero E, Pinotti G, et al. Patterns of survival in mantle cell lymphoma. Ann Oncol. 1995; 6:257-62. [PubMed: 7612491]

4. Romaguera JE, Fayad L, Rodriguez MA, et al. High rate of durable remissions after treatment of newly diagnosed aggressive mantle-cell lymphoma with rituximab plus hyper-CVAD alternating with rituximab plus high-dose methotrexate and cytarabine. J Clin Oncol. 2005; 23:7013-23. [PubMed: 16145068]

5. Eskelund CW, Kolstad A, Jerkeman M, et al. 15-year follow-up of the Second Nordic Mantle Cell Lymphoma trial (MCL2): prolonged remissions without survival plateau. Br J Haematol. 2016; 175:410-418. [PubMed: 27378674]

6. Hermine O, Hoster E, Walewski J, et al. Addition of high-dose cytarabine to immunochemotherapy before autologous stem-cell transplantation in patients aged 65 years or younger with mantle cell lymphoma (MCL Younger): a randomised, open-label, phase 3 trial of the European Mantle Cell Lymphoma Network. Lancet. 2016; 388:565-75. [PubMed: 27313086]

7. Kahl BS, Longo WL, Eickhoff JC, et al. Maintenance rituximab following induction chemoimmunotherapy may prolong progression-free survival in mantle cell lymphoma: a pilot study from the Wisconsin Oncology Network. Ann Oncol. 2006; 17:1418-23. [PubMed: 16766582]

8. Chang JE, Peterson C, Choi S, et al. VcR-CVAD induction chemotherapy followed by maintenance rituximab in mantle cell lymphoma: a Wisconsin Oncology Network study. Br J Haematol. 2011; 155:190-7. [PubMed: 21848883]

9. Chang JE, Li H, Smith MR, et al. Phase 2 study of VcR-CVAD with maintenance rituximab for untreated mantle cell lymphoma: an Eastern Cooperative Oncology Group study (E1405). Blood. 2014; 123:1665-73. [PubMed: 24458437]

10. National Cancer Institute. Common Terminology Criteria for Adverse Events v3.0. 2006. Available at: http://ctep.cancer.gov/forms/CTCAEv3.pdf

11. Cheson BD, Horning SJ, Coiffier B, et al. Report of an international workshop to standardize response criteria for non-Hodgkin's lymphomas. NCI Sponsored International Working Group. J Clin Oncol. 1999; 17:1244. [PubMed: 10561185] 
12. Geisler CH, Kolstad A, Laurell A, et al. Long-term progression-free survival of mantle cell lymphoma after intensive front-line immunochemotherapy with in vivo-purged stem cell rescue: a nonrandomized phase 2 multicenter study by the Nordic Lymphoma Group. Blood. 2008; 112:2687-93. [PubMed: 18625886]

13. Bernstein SH, Epner E, Unger JM, et al. A phase II multicenter trial of hyperCVAD MTX/Ara-C and rituximab in patients with previously untreated mantle cell lymphoma; SWOG 0213. Ann Oncol. 2013; 24:1587-93. [PubMed: 23504948]

14. Merli F, Luminari S, Ilariucci F, et al. Rituximab plus HyperCVAD alternating with high dose cytarabine and methotrexate for the initial treatment of patients with mantle cell lymphoma, a multicentre trial from Gruppo Italiano Studio Linfomi. Br J Haematol. 2012; 156:346-53. [PubMed: 22145911]

15. Kluin-Nelemans HC, Hoster E, Hermine O, et al. Treatment of older patients with mantle-cell lymphoma. N Engl J Med. 2012; 367:520-31. [PubMed: 22873532]

16. Kenkre VP, Long WL, Eickhoff JC, et al. Maintenance rituximab following induction chemoimmunotherapy for mantle cell lymphoma: long-term follow-up of a pilot study from the Wisconsin Oncology Network. Leuk Lymphoma. 2011; 52:1675-80. [PubMed: 21864042]

17. Le Gouill S, Thieblemont C, Oberic L, et al. Rituximab Maintenance after Autologous Stem Cell Transplantation Prolongs Survival in Younger Patients with Mantle Cell Lymphoma: Final Results of the Randomized Phase 3 LyMa Trial of the Lysa/Goelams Group. Blood. 2016; 128:145-145.

18. Romaguera JE, Fayad LE, Feng L, et al. Ten-year follow-up after intense chemoimmunotherapy with Rituximab-HyperCVAD alternating with Rituximab-high dose methotrexate/cytarabine (RMA) and without stem cell transplantation in patients with untreated aggressive mantle cell lymphoma. Br J Haematol. 2010; 150:200-8. [PubMed: 20528872]

19. Geisler CH, Kolstad A, Laurell A, et al. Nordic MCL2 trial update: six-year follow-up after intensive immunochemotherapy for untreated mantle cell lymphoma followed by BEAM or BEAC + autologous stem-cell support: still very long survival but late relapses do occur. Br J Haematol. 2012; 158:355-62. [PubMed: 22640180]

20. Maddocks K, Christian B, Jaglowski S, et al. A phase 1/1b study of rituximab, bendamustine, and ibrutinib in patients with untreated and relapsed/refractory non-Hodgkin lymphoma. Blood. 2015; 125:242-8. [PubMed: 25355819]

21. Johnson J, Kaplan LD, Koval G, et al. Minimal Residual Disease (MRD) Status Following Induction Chemo-Immunotherapy Predicts Progression-Free Survival In Mantle Cell Lymphoma (MCL): CALGB 50403 (Alliance). Blood. 2013; 122:3002-3002.

22. Pott C, Hoster E, Delfau-Larue MH, et al. Molecular remission is an independent predictor of clinical outcome in patients with mantle cell lymphoma after combined immunochemotherapy: a European MCL intergroup study. Blood. 2010; 115:3215-23. [PubMed: 20032498]

23. Pott C. Minimal residual disease detection in mantle cell lymphoma: technical aspects and clinical relevance. Semin Hematol. 2011; 48:172-84. [PubMed: 21782059] 
A

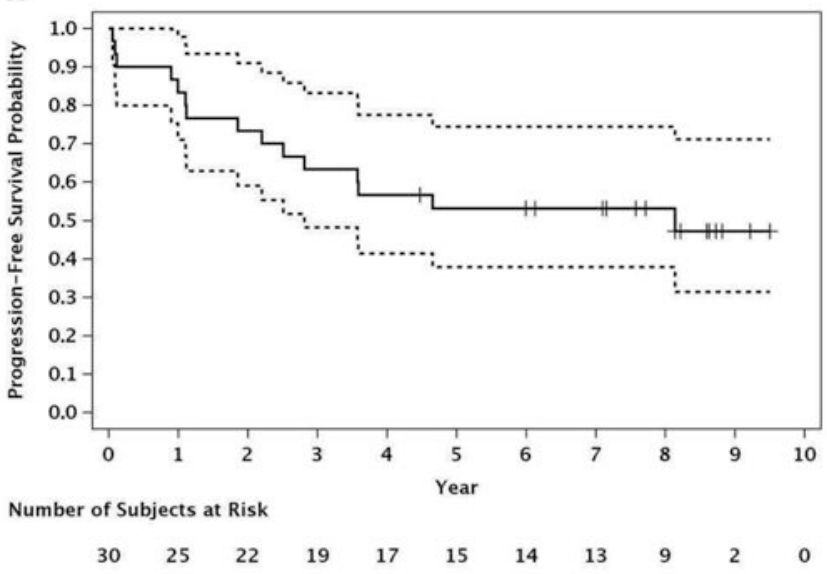

B

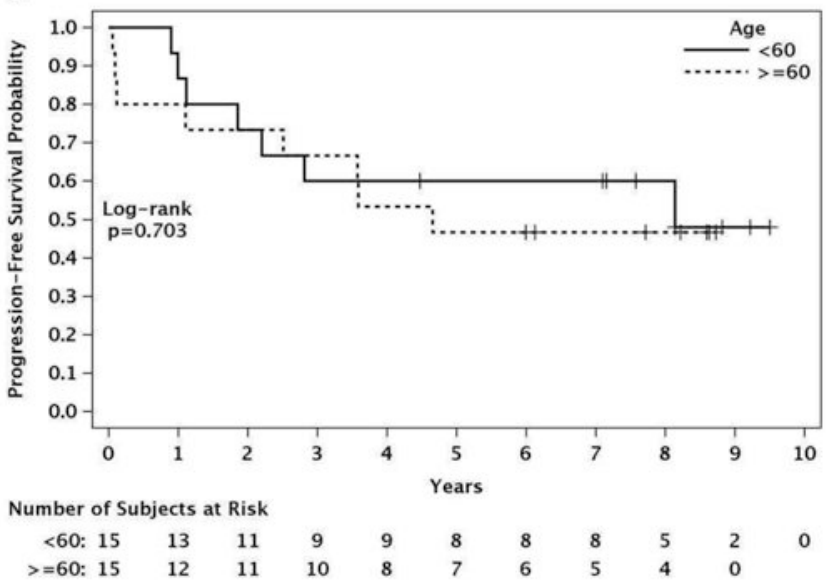

Figurre 1.

Progression-free survival.*

*The single event at 8 years was death from lung cancer (MCL in ongoing remission). 


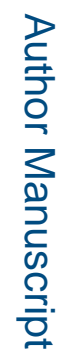

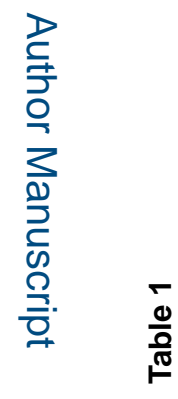

2)

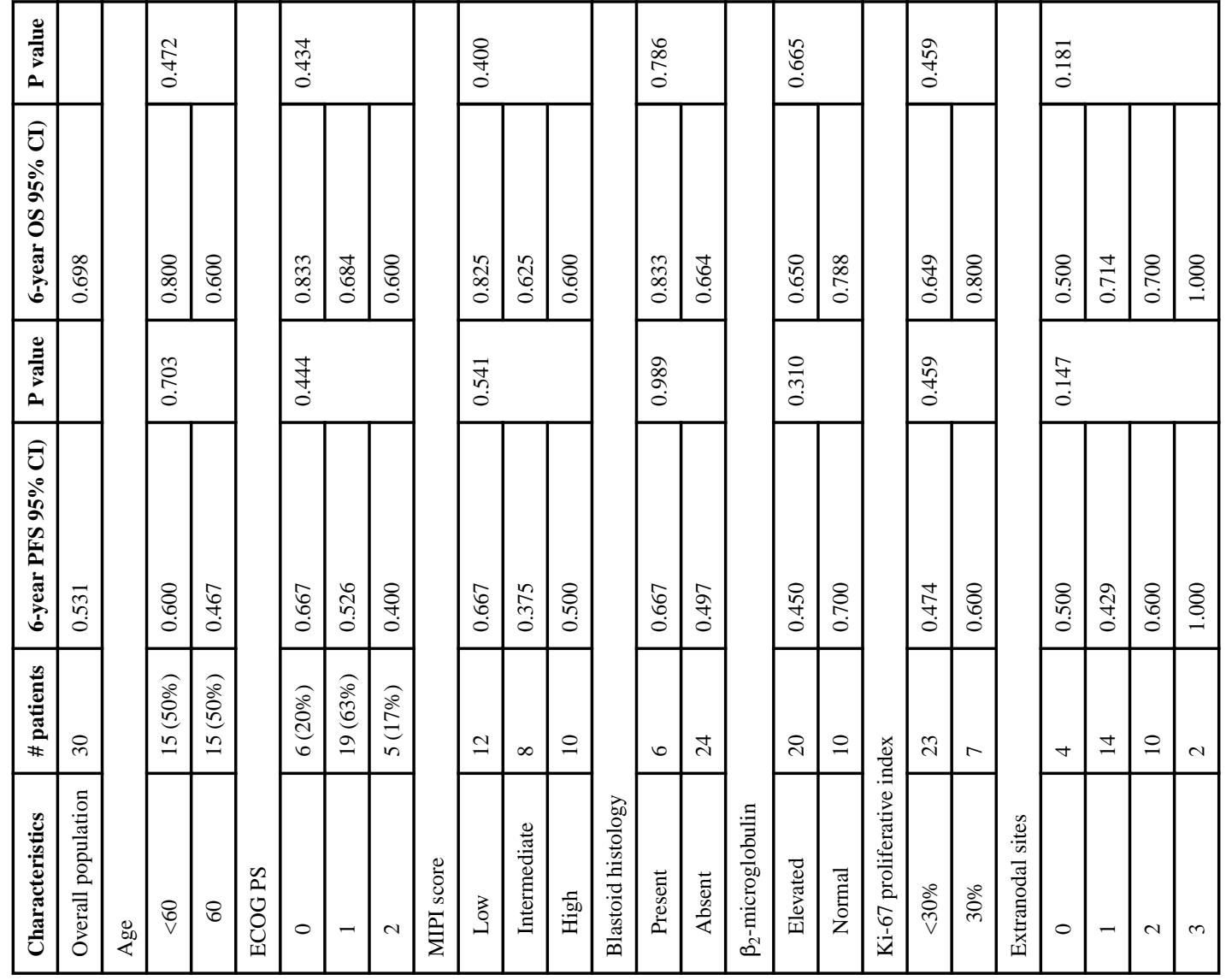

Clin Lymphoma Myeloma Leuk. Author manuscript; available in PMC 2018 April 12. 


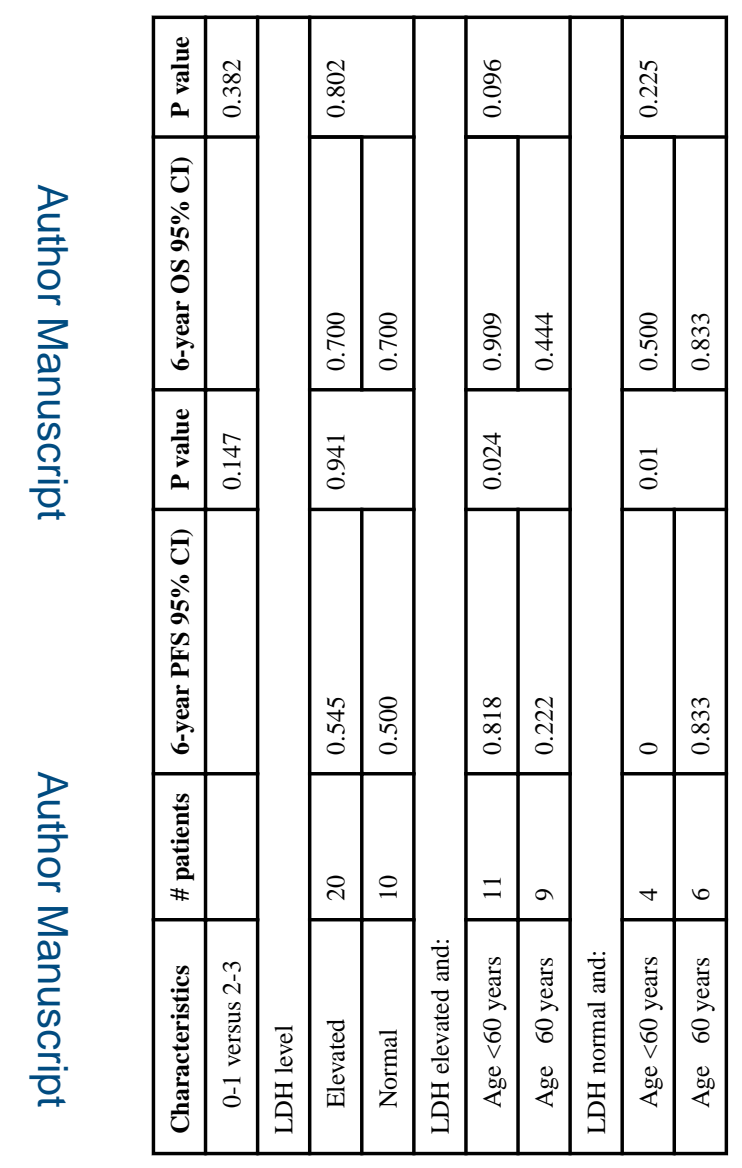

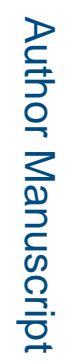

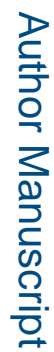


Table 2

Comparison of VcR-CVAD with other intensive induction and consolidation regimens

\begin{tabular}{|c|c|c|}
\hline & PFS & OS \\
\hline \multicolumn{3}{|c|}{$\begin{array}{l}\text { Intensive induction/consolidation } \\
\text { regimens }\end{array}$} \\
\hline $\begin{array}{l}\text { Nordic MCL2 }(n=160) \\
\text { R-maxi-CHOP/cytarabine }\end{array}$ & 6 -year $66 \%$ & 6 -year $70 \%$ \\
\hline $\begin{array}{l}\text { GELA }(n=60) \\
\text { R-CHOP/R-DHAP }\end{array}$ & Median 7 years & 5 -year $75 \%$ \\
\hline CALGB $59909(n=78)$ & 5 -year $56 \%$ & 5 -year $64 \%$ \\
\hline $\begin{array}{l}\text { R-hyper-CVAD (n=97) } \\
\text { Single-center }\end{array}$ & 3-year FFS 64\% & 3 -year $82 \%$ \\
\hline $\begin{array}{l}\text { R-hyper-CVAD (n=60) } \\
\text { GISL }\end{array}$ & 5-year PFS 61\% & 5 -year $73 \%$ \\
\hline $\begin{array}{l}\text { R-hyper-CVAD }(n=49) \\
\text { SWOG } 0213\end{array}$ & Median 4.8 years & Median 6.8 years \\
\hline \multicolumn{3}{|l|}{$\begin{array}{l}\text { VcR-CVAD induction } \\
\text { maintenance rituximab }\end{array}$} \\
\hline $\begin{array}{l}\text { WON }(n=30) \\
5 \text { years MR }\end{array}$ & $\begin{array}{l}\text { 6-year PFS } 53 \% \\
6 \text {-year PFS } 60 \% \text { for age }<60\end{array}$ & 6 -year $70 \%$ \\
\hline $\begin{array}{l}\text { E1405 }(\mathrm{n}=75) \\
2 \text { years MR } \\
22 \text { received received ASCT }\end{array}$ & $\begin{array}{l}\text { 3-year PFS 72\% } \\
\text { 3-year PFS } 67 \% \text { for MR-treated patients }\end{array}$ & 3-year $88 \%$ \\
\hline
\end{tabular}

